\title{
DIVERSIDAD ORTOPTEROLÓGICA DE LA RESERVA TERRITORIAL SURESTE DE LA CIUDAD UNIVERSITARIA (UNAM)
}

\section{Iván CASTELLANOS-VARGAS ${ }^{1}$, PATricia L. GARCÍA-GARCÍA ${ }^{2,3}$ y Zenón CANO-SANTANA ${ }^{1}$}

\author{
${ }^{1}$ Grupo de Interacciones y Procesos Ecológicos. Departamento de Ecología y Recursos Naturales, Facultad de \\ Ciencias, Universidad Nacional Autónoma de México. México Distrito Federal C.P. 04510. \\ <icv@ciencias.unam.mx> \\ ${ }^{2}$ Instituto de Fitosanidad, Colegio de Postgraduados. Km 36.5 Carr. México-Texcoco, Montecillo, Estado de \\ México C.P. 56230. \\ ${ }^{3}$ Adscripción académica actual: Red de Ecología Funcional. Instituto de Ecología A.C., Xalapa, Veracruz. \\ Carretera antigua a Coatepec 351, El Haya C.P. 91070.
}

Recibido: 27/08/2014; aceptado: 21/11/2014

\begin{abstract}
Castellanos-Vargas, I., García-García, P. L. \& Cano-Santana, Z. 2015. Diversidad ortopterológica de la Reserva Territorial Sureste de la Ciudad Universitaria (UNAM). Acta Zoológica Mexicana (n. s.), 31(1): 97-108.
\end{abstract}

RESUMEN. El crecimiento constante de la población humana hace que la mancha urbana extienda sus límites hacia las reservas ecológicas reduciendo el tamaño de éstas, generando importantes efectos de borde que acentúan la fragmentación del paisaje y que potencializan la pérdida de continuidad de la cubierta vegetal.

Las reservas ecológicas, así como las áreas verdes urbanas (representadas por parques y jardines) son hábitats relictuales para una gran diversidad de especies que sobreviven dentro de la matriz urbana. La Reserva Ecológica del Pedregal de San Ángel de Ciudad Universitaria (REPSA-CU) es la más importante en el suroeste del Distrito Federal debido a que abarca 237.3 hectáreas y en ella se conservan 377 especies de plantas vasculares, 180 especies de vertebrados nativos y por lo menos 817 especies de artrópodos, de las cuales 26 son ortópteros con una importante posición dentro de la cadena trófica y son determinantes del funcionamiento del ecosistema.

Los objetivos de este trabajo fueron: 1. Conocer las especies de ortópteros presentes en la Reserva Territorial Sureste de la Ciudad Universitaria de la Universidad Nacional Autónoma de México (RTSE), mediante la elaboración de un inventario biológico; 2. Comparar los atributos de la comunidad ortopterológica (abundancia, diversidad; así como la densidad de individuos) que habitan en la RTSE en relación con los ortópteros de la Zona Núcleo Suroriente (ZNSO) de la REPSACU y finalmente, 3. Discutir sobre el valor ambiental de la RTSE como Zona de Amortiguamiento Ambiental para la REPSA-CU.

La abundancia relativa por especie no varió significativamente entre la RTSE y la zona núcleo revisada. En la RTSE se encontraron cinco especies de ortópteros, la especie que registró mayor abundancia fue Sphenarium purpurascens purpurascens Charpentier, 1842 (Pyrgomorphidae) seguida en orden de importancia por Phoetaliotes nebrascensis (Thomas, 1872) (Acrididae). Destaca la presencia de Conocephalus (Xiphidion) ictus (Scudder, 1875) (Tettigoniidae) como un nuevo registro taxonómico para la zona. Se discute que la RTSE difiere en cuanto a diversidad ortopterológica con la ZNSO de la REPSA-CU y argumentamos su importancia para la conservación de vida silvestre; así como la importancia que poseen los ortópteros de acuerdo con su posición en la cadena trófica para el funcionamiento del ecosistema.

Palabras clave: Conocephalus (Xiphidion) ictus, fragmentación del hábitat, ortopterofauna, Reserva Ecológica del Pedregal de San Ángel, Sphenarium purpurascens purpurascens, urbanización.
Castellanos-Vargas, I., García-García, P. L. \& Cano-Santana, Z. 2015. Orthopterological diversity in the southeastern of Ciudad Universitaria (UNAM). Acta Zoológica Mexicana (n. s.), 31(1): 97-108.

ABSTRACT. The steady growth of human population causes that the urban area extends its limits to ecological reserves reducing the size of these, generating significant edge effects that accentuate the landscape fragmentation and increase the loss of continuity from vegetation cover.

Ecological reserves and the urban green areas (represented by parks and gardens) are relict habitats for many species that survive in the urban matrix. The Pedregal de San Angel Ecological Reserve at Ciudad Universitaria (PSAER-CU) is the most important in the southwest of Mexico City because it embodies 237.3 hectares and possesses 377 species of vascular plants, 180 species of native vertebrates and preserves at least 817 arthropod species, out of which 26 are orthopterans, the latter play an important role in the food chain and are determinants of ecosystem functioning.

This study aimed to: 1 . Elaborate a check list of the Orthoptera species that survive in the Southeastern Territorial Reserve from Ciudad Universitaria (SETR); 2. Compare the attributes (abundance, diversity and density) of the Orthoptera community that inhabit the SETR with respect to the Orthoptera species occurring at the Southeast Core Zone at PSAER; 3. Discuss the environmental value of the SETR as Environmental Buffer Zone for PSAER.

The relative abundance of each species did not differ significantly between the SETR and the Core Zone. We found five Orthoptera species at SETR, the species with greatest abundance was Sphenarium purpurascens purpurascens Charpentier, 1842 Pyrgomorphidae, followed by Phoetaliotes nebrascensis (Thomas, 1872) Acrididae. The presence of Conocephalus (Xiphidion) ictus (Scudder, 1875) Tettigoniidae represents a new taxonomic record for the study area.

We discuss that the SETR differs on orthopterological diversity from the Southeast Core Zone at PSAER and argue its importance for wildlife conservation; as well as the importance that the Orthoptera possess according to their position in the food chain and why they are determinants of ecosystem functioning.

Key words: Conocephalus (Xiphidion) ictus, habitat fragmentation, Orthoptera, Pedregal de San Angel Ecological Reserve, Sphenarium purpurascens purpurascens, urbanization. 


\section{INTRODUCCIÓN}

El centro de México es un sitio potencial para la especiación de ortopteroides debido a las condiciones biogeográficas que prevalecen sobre el territorio nacional (Barrientos-Lozano 2004), y aún en el siglo XXI se desconoce cuántas especies o subespecies pueden ser endémicas. La realización de estudios ortopterofaunisticos esclarecerá el valor de diversidad beta para varias localidades que desarrollan sus comunidades vegetales con límites difusos y en mosaicos (Zepeda 1961, Whittaker 1965, Rzedowski 1978, Rzedowski \& Rzedowski 1979).

Los ortopteroides son uno de los grupos con mayor diversidad de la clase Hexapoda, estimándose a nivel mundial alrededor de 24557 especies de ortópteros distribuidas principalmente en las regiones tropicales del planeta con clima cálido (Capinera et al. 2004). En México se han descrito alrededor de 650 especies (Eades et al. 2013), y para el Distrito Federal se considera la presencia de 47 especies agrupadas en 40 géneros, ubicados en 143 subfamilias, dentro de siete Superfamilias (E. Rivera-García, com. pers.). El 53.25\% de los registros del Distrito Federal corresponden a la superfamilia Acridoidea. Estudios realizados por Wood \& Samways (1991), Solomon \& Samways (2006) y Cherrill (2010) señalan que existen especies indicadoras de perturbaciones en el ambiente principalmente debidas a la fragmentación y la urbanización.

Las reservas ecológicas y las áreas verdes urbanas (representadas por parques y jardines) son hábitats relictuales para una gran diversidad de especies que sobreviven dentro de la matriz urbana (Gaston et al. 2005, Angold et al. 2006). El crecimiento constante de la población humana hace que la mancha urbana extienda sus límites hacia las reservas ecológicas reduciendo el tamaño de éstas, generando importantes efectos de borde que acentúan la fragmentación del paisaje y que potencializan la pérdida de continuidad de la cubierta vegetal, así como las extinciones locales de algunas especies (Knight \& Holt 2005, Angold et al. 2006, Ortega-Álvarez \& MacGregor-Fors 2009, Cherrill 2010).

La Reserva Ecológica del Pedregal de San Ángel de Ciudad Universitaria (REPSA-CU) es la más importante en el suroeste del Distrito Federal debido a que abarca 237.3 ha y en ella se conservan 377 especies de plantas vasculares, 180 especies de vertebrados nativos y por lo menos 817 especies de artrópodos, de las cuales 26 son ortópteros con una posición importante dentro de la cadena trófica, que los hace determinantes del funcionamiento del ecosistema (Márquez-Mayaudón 1968, Álvarez-Sánchez et al. 1982, Valiente-Banuet \& de Luna 1990, Castillo-Argüero et al. 2004, 2007, Hortelano-Moncada et al. 2009, Rueda \& Cano 2009).
Los objetivos de este trabajo fueron: 1. Conocer las especies de ortópteros presentes en la Reserva Territorial Sureste de la Ciudad Universitaria de la Universidad Nacional Autónoma de México (RTSE), mediante la elaboración de un inventario biológico; 2. Comparar los atributos de la comunidad ortopterológica (abundancia, riqueza y diversidad de especies); así como la densidad de la RTSE con los ortópteros de la Zona Núcleo Suroriente (ZNSO) de la REPSA-CU y 3. Discutir el valor de la RTSE como Zona de Amortiguamiento Ambiental para la REPSA-CU.

\section{MATERIALES Y MÉTODOS}

Área de estudio. La Ciudad Universitaria se ubica en el Suroeste de la Ciudad de México dentro de la Delegación Coyoacán y ocupa un terreno cuya superficie es de aproximadamente 712 ha (Fig. 1). Los terrenos bajo la administración de Universidad Nacional Autónoma de México se clasifican en tres categorías: 1. Reserva Territorial Urbana (RTU), 2. Reserva Ecológica del Pedregal de San Ángel (REPSA-CU) y 3. Zonas de Amortiguamiento de la REPSA-CU.

La RTU comprende un conjunto de terrenos cuyas superficies son variables y algunos albergan importantes fragmentos relictuales del basalto y la flora del Pedregal de San Ángel, por lo que tienen un valor ambiental aún no estimado (Suárez et al. 2011). En estas zonas, se proyecta el crecimiento de la infraestructura civil y urbana de las diferentes dependencias de la Universidad, que acentúan el deterioro del paisaje provocando una matriz urbana, originada por la fragmentación, la reducción y eventual pérdida de la comunidad biótica emblemática de esta zona de la Ciudad de México (Suárez et al. 2011).

Por su parte, la REPSA-CU ocupa la tercera parte del campus Universitario (237.3 ha) y alberga un importante remanente de matorral xerófilo cuyo elemento florístico emblemático es Pittocaulon [Senecio] praecox (Cav.) H.Rob. \& Brettell. Esta comunidad vegetal es la mejor representada de las nueve que se asentaron sobre el sustrato basáltico del derrame del volcán Xitle y cubría cerca de 80 km² (Rzedowski 1954, Carrillo-Trueba 1995). Según datos de Camarena-Berruecos (2010), actualmente los afloramientos rocosos con remanentes de esta comunidad vegetal se estiman en $3.65 \mathrm{~km}^{2}$ y se encuentran dispersos en las delegaciones Álvaro Obregón, Coyoacán y Tlalpan.

El clima es templado subhúmedo con lluvias en verano; presenta estacionalidad marcada, la época de lluvias comprende de junio a octubre mientras que la temporada seca comprende el resto del año (noviembre a mayo); la temperatura media anual es de $15.6^{\circ} \mathrm{C}$ y la precipitación media anual es de 833 mm (Lot \& Camarena 2009). 


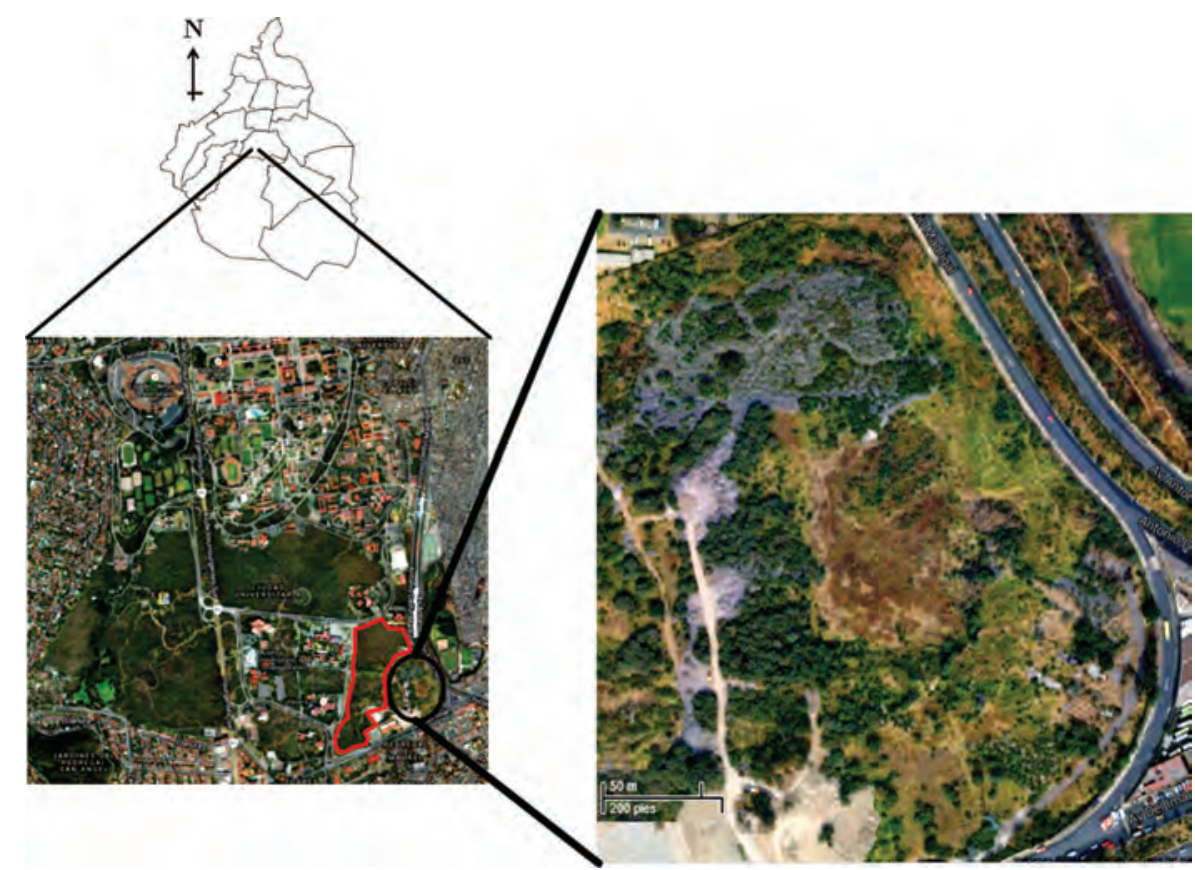

Figura 1. Localización de la Reserva Territorial Sureste (RTSE) en el campus de la Ciudad Universitaria. Hacia la izquierda del círculo inferior, se muestra el contorno de la Zona Núcleo Suroriente (ZNSO) de la Reserva Ecológica del Pedregal de San Ángel (REPSA-CU).

De acuerdo con el trabajo de Peralta \& Prado (2009), la REPSA-CU se compone de tres Zonas Núcleo (ZN) que en conjunto abarcan una extensión de 171.3 ha; así como por 13 Zonas de Amortiguamiento (ZA) que comprenden 66 ha. El sustrato de las ZN está representado por un basalto volcánico de olivino gris obscuro con microcristales y es altamente heterogéneo, su espesor varía en algunos lugares de $50 \mathrm{~cm}$ a $10 \mathrm{~m}$; las diferencias de nivel en el terreno originaron la formación de grietas, hondonadas, sitios planos y promontorios rocosos; en ellos la acumulación del suelo es diferencial y en general se caracteriza por ser escaso y poco profundo (Rzedowski 1954, Enciso de la Vega 1979, Álvarez-Sánchez et al. 1982, Cano-Santana \& Meave 1996, Santibáñez-Andrade 2005, Santibáñez-Andrade et al. 2009). Por su parte, en las ZA es frecuente observar cierto grado de perturbación del suelo representada por la presencia de depósitos de basura inorgánica y restos de materiales de construcción (cascajo) que se acumulan sobre el sustrato basáltico. En estas zonas los materiales edáficos no consolidados son invadidos por vegetación ruderal como el pasto africano Pennisetum clandestinum, la higuerilla Ricinus communis y las ornamentales Tropaeolum majus y Leonotis nepetifolia.

Delimitaciones de las comunidades vegetales para el muestreo. La Zona de Reserva Territorial Sureste (RTSE) está ubicada en la esquina de las avenidas Delfín Madrigal e IMÁN. Este sitio tiene una extensión aproximada de 10.5 ha y colinda al poniente con la Zona Núcleo
Suroriente (ZNSO) de la REPSA-CU (Fig. 1).

En octubre de 2005, época de mayor diversidad vegetal y entomológica se delimitaron cuatro diferentes tipos de comunidades fisonómicas en la RTSE y en la ZNSO: 1) Pastizal. Dominado por el pasto Pennisetum clandestinum que crece exitosamente en suelos perturbados (Castellanos-Vargas 2001). 2) Pradera mixta. Con presencia de gramíneas acompañadas por Cosmos bipinnatus, en estos sitios $P$. clandestinum esta ausente o es inconspicuo. 3) Pedregal con matorral xerófilo que se desarrolla sobre los sitios donde existen afloramientos de basalto y permite el asentamiento de Pittocaulon praecox, Tagetes lunulata, Muhlenbergia robusta, Wigandia urens, Opuntia tomentosa y Manfreda scabra entre otras. A pesar de su escasa presencia en la localidad, este tipo de vegetación se puede encontrar representado en dos formas fisonómicas diferentes: i) Matorral xerófilo abierto, dominado por la presencia de hierbas y ii) Matorral xerófilo cerrado y finalmente, 4) Bosquetes de tepozán dominados por Buddleia cordata. Los árboles de este sitio tienen un follaje distribuido en un dosel monoestratificado cercano a $3 \mathrm{~m}$ de altura y en el sotobosque existen algunas gramíneas como el pasto $P$. clandestinum.

Muestreo de ortópteros. A fin de comparar la abundancia y la riqueza de especies, así como la densidad de individuos que habitan en la RTSE y en la ZNSO, en octubre de 2005 se muestrearon mediante redes entomológicas de golpeo 12 cuadros de $1 \times 1 \mathrm{~m}$, elegidos al azar en cada tipo de vegetación. Los muestreos fueron realizados entre 
las 8:30 y las 16:30 h. Los ortópteros se depositaron en cámaras letales de acetato de etilo al 60\%. Este material entomológico se etiquetó y trasladó al laboratorio de Ecología de Artrópodos Terrestres de la Facultad de Ciencias de la UNAM donde se separó y montó de acuerdo con las especificaciones correspondientes al orden Orthoptera.

Para la determinación taxonómica del material se utilizó el trabajo de Márquez-Mayaudón (1968) debido a la afinidad del sitio de estudio con el Pedregal de San Ángel original, así como las claves contenidas en las memorias del curso-taller "Identificación y Manejo del Chapulín” publicado por el Comité Estatal de Sanidad Vegetal del Estado de Hidalgo (CESAVEH 2004) y el catálogo fotográfico de Ortópteros del Pedregal de San Ángel del M. en C. Iván Castellanos-Vargas (datos no publ.).

Con la identidad taxonómica de los ejemplares se elaboró un inventario biológico para la Zona Sureste de la Ciudad Universitaria (UNAM) de acuerdo con los lineamientos de Eades et al. (2013). Todo el material ortopterológico fue depositado en la Colección Nacional de Insectos del Instituto de Biología de la UNAM (CNINIBUNAM), para consultas posteriores.

Análisis estadístico. A fin de establecer los efectos de las dos zonas de muestreo (RTSE y ZNSO) y de las cuatro comunidades fisonómicas de vegetación sobre la abundancia y la riqueza de especies, así como la densidad de individuos, se realizaron Análisis de Varianza de dos factores (ANdeVA II). Previamente a la realización de este análisis, se comprobó la normalidad de la distribución de los datos mediante una prueba de $z$.

A fin de lograr la normalización de las distribuciones de la densidad, la abundancia y la riqueza específica de los ortópteros, los datos se transformaron como $x^{\prime}=\sqrt{ } x+0.5$ por tratarse de variables discretas (Sokal \& Rohlf 1995).

Con la finalidad de conocer las diferencias significativas de los atributos de la comunidad ortopterológica y densidad entre los sitios (RTSE y ZNSO) así como entre los tipos de vegetación que cada uno ostenta, se realizaron pruebas de Tukey de comparación múltiple de medias para muestras con tamaños similares (Sokal \& Rohlf 1995).

Con la finalidad de comparar la composición de las comunidades mediante la abundancia relativa, se calculó una distribución $\chi^{2}$ calculando los valores esperados para cada especie mediante una tabla de contingencia que consideró a los dos sitios (RTSE y ZNSO) $\times$ la abundancia de cada especie (Sokal \& Rohlf 1995). A fin de comparar el valor de diversidad entre sitios así como entre las comunidades vegetales de ambos, se calculó el índice de Shannon-Wiener $\left(H^{\prime}\right)$ considerando una base logarítmica decimal y se aplicaron pruebas de $t$ de Student para muestras independientes especialmente modificadas para índices de diversidad (Zar 2010). Para establecer las diferencias significativas entre pares de comparaciones, se aplicaron correcciones de Bonferroni, para evitar cometer un error de tipo I (Sokal \& Rohlf 1995, Zar 2010).

Todos los análisis estadísticos se realizaron con el paquete STATISTICA v.8.0 (StatSoft 2008) y se obtuvieron bajo la supervisión técnica del personal del Laboratorio Ecología de Artrópodos Terrestres del Departamento de Ecología y Recursos Naturales de la Facultad de Ciencias de la Universidad Nacional Autónoma de México.

\section{RESULTADOS}

Diversidad taxonómica. La diversidad ortopterológica del Sureste de Ciudad Universitaria estuvo representada por seis especies correspondientes a cinco subfamilias. La familia mejor representada fue Acrididae con cuatro especies (Cuadro 1). En la RTSE se encontraron cinco especies pertenecientes a tres familias, mientras que por su parte en la ZNSO sólo se encontró una especie adicional Trimerotropis pallidipennis (Cuadro 1).

En ambos sitios se registró a Conocephalus (Xiphidion) ictus (Scudder, 1875) (Tettigoniidae: Conocephalinae) el cual constituye un nuevo registro taxonómico para la REPSA-CU (Cuadro 1).

Composición de especies. En octubre de 2005 se colectaron en ambos sitios un total de 323 ejemplares de ortópteros (173 en la RTSE y 150 en la ZNSO). La composición porcentual de las especies no varió significativamente entre ambas zonas $\left(\chi^{2}=8.249\right.$ g.l. $\left.=4, P=0.140\right)$. La especie más conspicua en ambos sitios fue Sphenarium purpurascens purpurascens con 121 ejemplares (70\%) en la RTSE y 95 ejemplares (64\%) en la ZNSO, seguida en orden de importancia por Phoetaliotes nebrascensis con 19 ejemplares (11\%) en la RTSE y Achurum sumichrasti con 24 ejemplares (16\%) en la ZNSO (Fig. 2). Las especies con menor abundancia relativa en la comunidad ortopterológica de la ZNSO fueron Melanoplus gladstoni y $T$. pallidipennis pues entre ambas aportaron el $2 \%$ de la composición de especies (Fig. 2).

El pastizal de la ZNSO fue el tipo de vegetación donde se registró el total de las especies colectadas ( $\mathrm{N}=6$ especies). Por su parte, en el pastizal y la pradera de la RTSE, así como la pradera de la ZNSO se registró el 67\% de las especies, pues en cada una se capturaron 4 especies (Cuadro 2). Los sitios donde se encontró la menor riqueza específica fueron los matorrales cerrados y los bosquetes de tepozán.

Densidad de ortópteros. La densidad de ortópteros no fue afectada significativamente por el tipo de sitio $\left(F_{14}\right.$ $=0.608, P=0.479$ ), por lo que los valores promedio entre RTSE y ZNSO (2.88 ortópteros $/ \mathrm{m}^{2} \pm$ error estándar 
Cuadro 1. Listado de las especies de ortópteros registradas en octubre de 2005 en el Sureste de la Ciudad Universitaria (UNAM). La "X” denota en cada caso la presencia de dicha especie en la zona correspondiente. RTSE: Reserva Territorial Sureste. ZNSO: Zona Núcleo Suroriente de la REPSA-CU. La presencia de la especie Conocephalus (Xiphidion) ictus (Scudder, 1875) constituye un nuevo registro para la zona de estudio.

\begin{tabular}{|c|c|c|}
\hline Suborden: Ensifera & RTSE & ZNSO \\
\hline \multicolumn{3}{|l|}{ Superfamilia: Tettigonioidea Krauss, 1902} \\
\hline \multicolumn{3}{|l|}{ Familia: Tettigoniidae Krauss, 1902} \\
\hline \multicolumn{3}{|l|}{ Tribu: Conocephalini Burmeister, 1838} \\
\hline \multicolumn{3}{|l|}{ Género: Conocephalus Thunberg, 1815} \\
\hline
\end{tabular}

Superfamilia: Pyrgomorphoidea Brunner von Wattenwyl, 1882

Familia: Pyrgomorphidae Brunner von Wattenwyl, 1882

Subfamilia: Pyrgomorphinae Brunner von Wattenwyl, 1882

Tribu: Sphenariini Bolívar, 1884

Género: Sphenarium Charpentier, 1842

Especie y subespecie: Sphenarium purpurascens purpurascens Charpentier, 1842 Det. Iván

Castellanos-Vargas, 2005

Superfamilia: Acridoidea MacLeay, 1821

Familia: Acrididae MacLeay, 1821

Subfamilia: Melanoplinae Scudder, 1897

Tribu: Melanoplini Scudder, 1897

Género: Melanoplus Stål, 1873

Especie: Melanoplus gladstoni Scudder, 1897 Det. Iván Castellanos-Vargas, 2005

$\mathrm{X}$

Especie: Phoetaliotes nebrascensis (Thomas, 1872) Det. Iván Castellanos-Vargas, 2005

Subfamilia: Gomphocerinae Fieber, 1853

Tribu: Mermiriini Brunner von Wattenwyl, 1893

Género: Achurum Saussure, 1861

Especie: Achurum sumichrasti (Saussure, 1861) Det. Iván Castellanos-Vargas, 2005

Subfamilia: Oedipodinae Walker, 1871

Tribu: Trimerotropini Blatchley, 1920

Género: Trimerotropis Stål, 1873

Especie: Trimerotropis pallidipennis (Burmeister, 1838) Det. Iván Castellanos-Vargas, 2005
$\mathrm{X}$

$\mathrm{X}$

$\mathrm{X}$

$\mathrm{X}$

$\mathrm{X}$

X $\quad X$

$\mathrm{X}$
0.39 y 2.50 ortópteros $/ \mathrm{m}^{2} \pm$ e.e. 0.42 ; respectivamente) no difirieron. No obstante, esta misma prueba señaló que los tipos de vegetación, así como su interacción con ambos sitios sí afectaron significativamente a la densidad de ortópteros $\left(F_{4,350}=140.307, P<0.0001\right.$ y $F_{4,350}=6.637$, $P<0.0001$; respectivamente). La prueba de Tukey determinó que los sitios con vegetación herbácea (pastizales y praderas), así como los matorrales con fisonomía abierta registraron valores de densidad significativamente más alta (de entre 8.0 a 2.1 ortópteros $/ \mathrm{m}^{2}$ ) en comparación con los bosquetes de tepozán y los matorrales xerófilos con aspecto cerrado (Fig. 3).

Diversidad cuantitativa. En la RTSE el índice de Shannon-Wiener ( $H^{\prime}$ ) fue 0.438 , mientras que en la ZNSO el índice fue 0.488 . De acuerdo con la prueba de $t$ de Student modificada para $H$ ', estos valores son significativamente diferentes entre si $(t=3.192$ g.l. $=701, P=0.0014)$. Cabe aclarar que los grados de libertad se calcularon de acuerdo con el método propuesto por Zar (2010). Al ajustar el nivel de significancia mediante la prueba de Bonferroni 


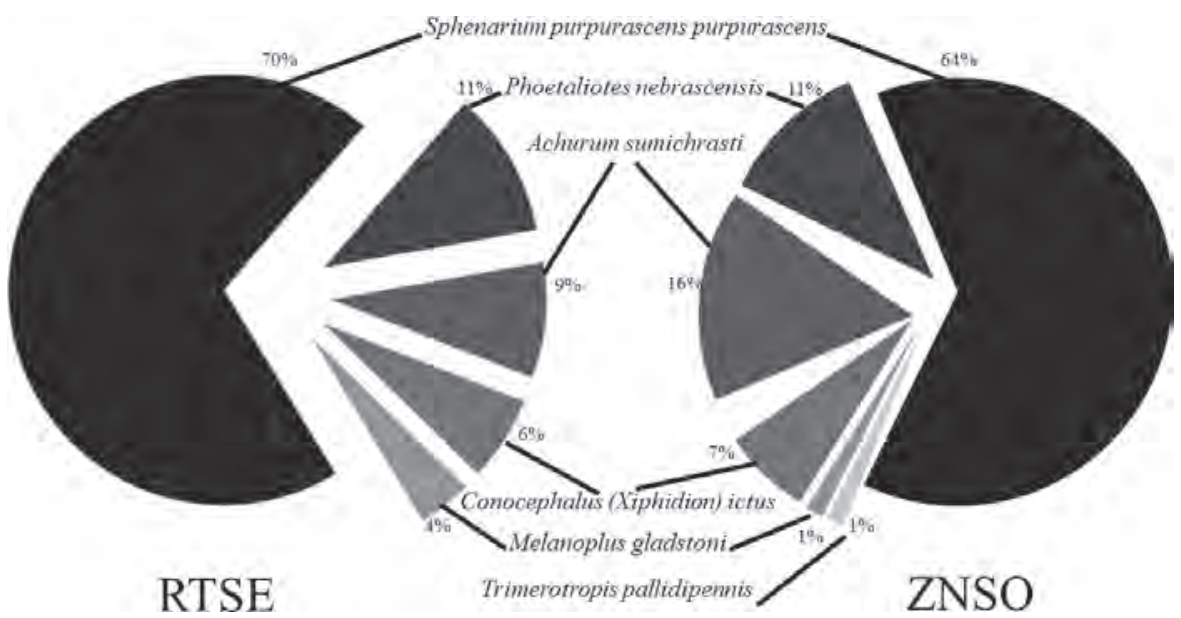

Figura 2. Abundancia relativa de las comunidades de ortópteros de la Reserva Territorial Sureste (RTSE) y de la Zona Núcleo Suroriente (ZNSO). Trimerotropis pallidipennis (Acrididae: Oedipodinae) sólo se encontró en la ZNSO de la Reserva Ecológica del Pedregal de San Ángel de Ciudad Universitaria (REPSA-CU).

Cuadro 2. Registros de presencia/ausencia (1 y 0, respectivamente) de las especies de ortópteros registradas en octubre de 2005 en cada una de las comunidades vegetales del Sureste de la Ciudad Universitaria (UNAM). RTSE: Reserva Territorial Sureste. ZNSO: Zona Núcleo Suroriente de la REPSA-CU.

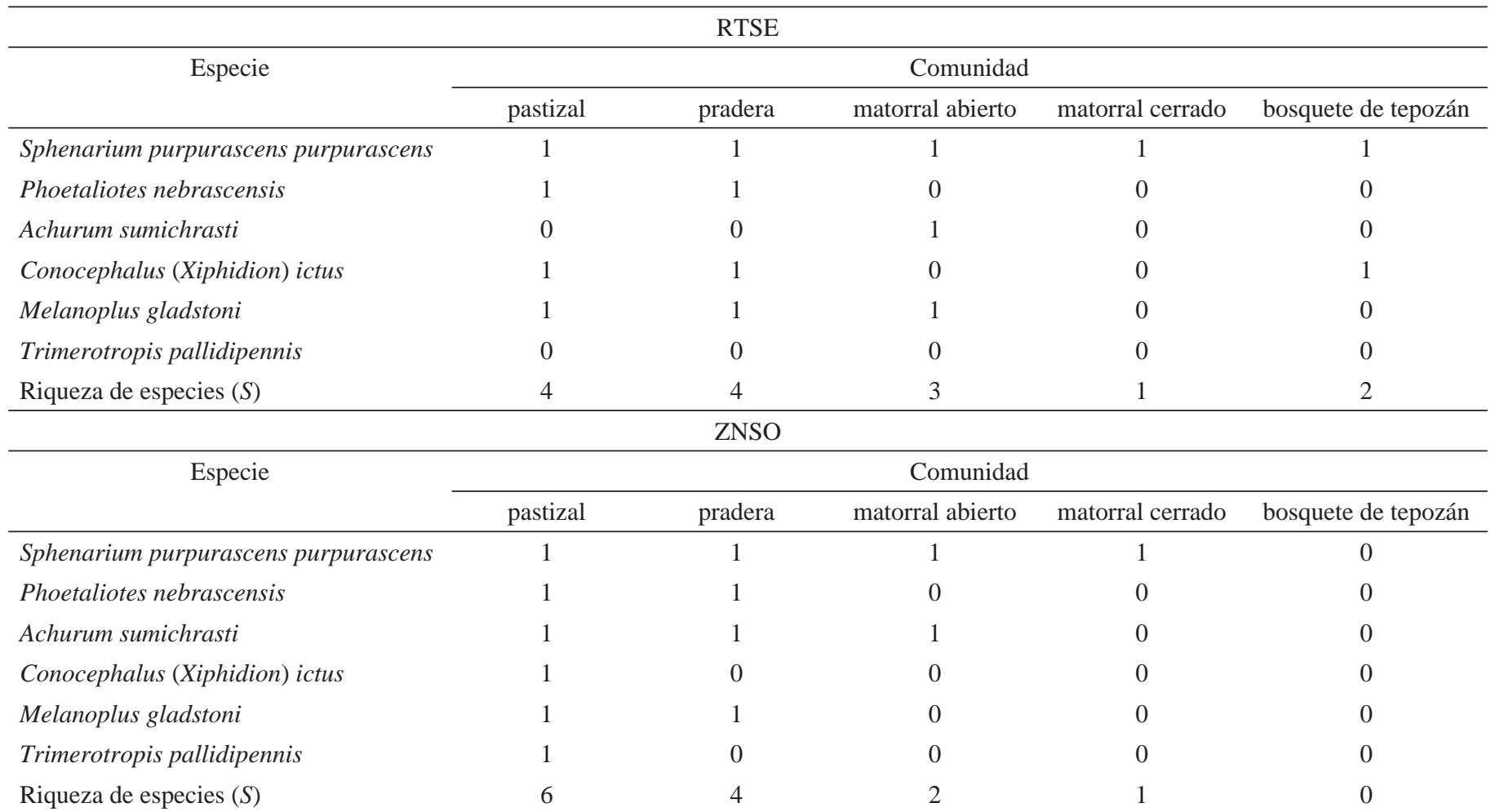

para las comparaciones múltiples de $H^{\prime}$ (por sitios y comunidades vegetales) se encontró que la variación de la diversidad guardó un orden de importancia similar al que se reportó para la densidad de ortópteros en este mismo estudio. Los sitios con vegetación herbácea (pastizales y praderas), así como los matorrales con fisonomía abierta registraron valores de diversidad significativamente más alta en comparación con los bosquetes de tepozán y los matorrales xerófilos con aspecto cerrado (Fig. 4).

\section{DISCUSIÓN}

Algunos datos de Carrillo-Trueba (1995); Peralta \& Prado (2009) y de Lot \& Camarena (2009) sugieren que la ex- 


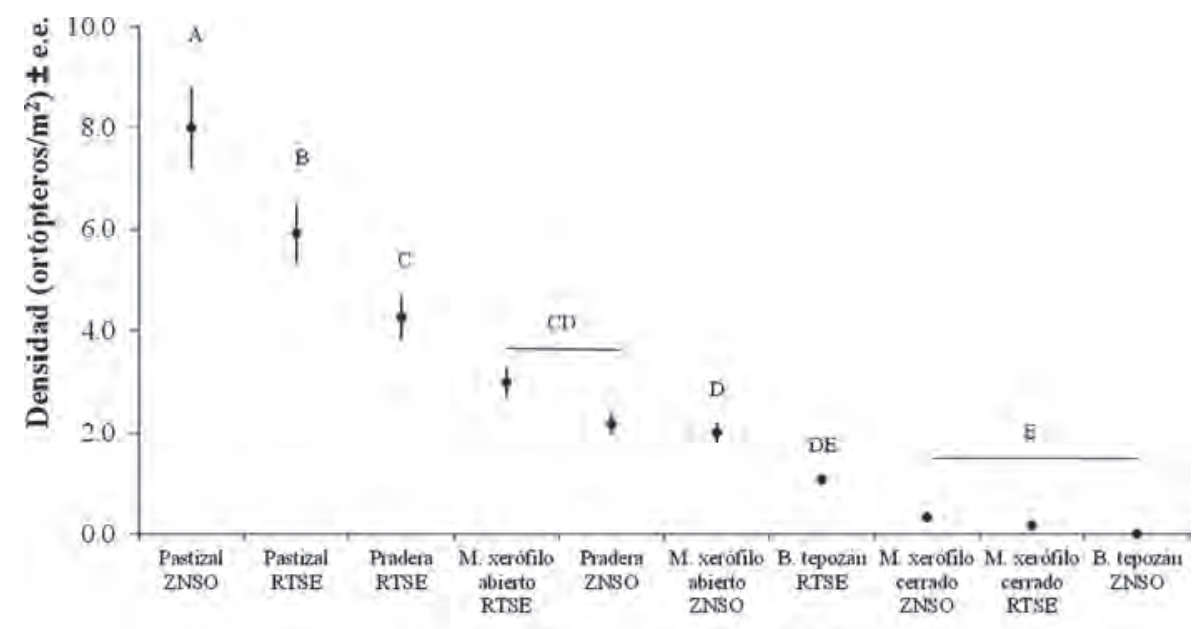

Sitios y comunidades vegetales

Figura 3. Variación de la densidad promedio de ortópteros ( \pm error estándar) entre los tipos de vegetación de la Reserva Territorial Sureste (RTSE) y los de la Zona Núcleo Suroriente (ZNSO). Las letras diferentes en cada caso denotan diferencias significativas (con $P<0.05)$ según la prueba de Tukey.

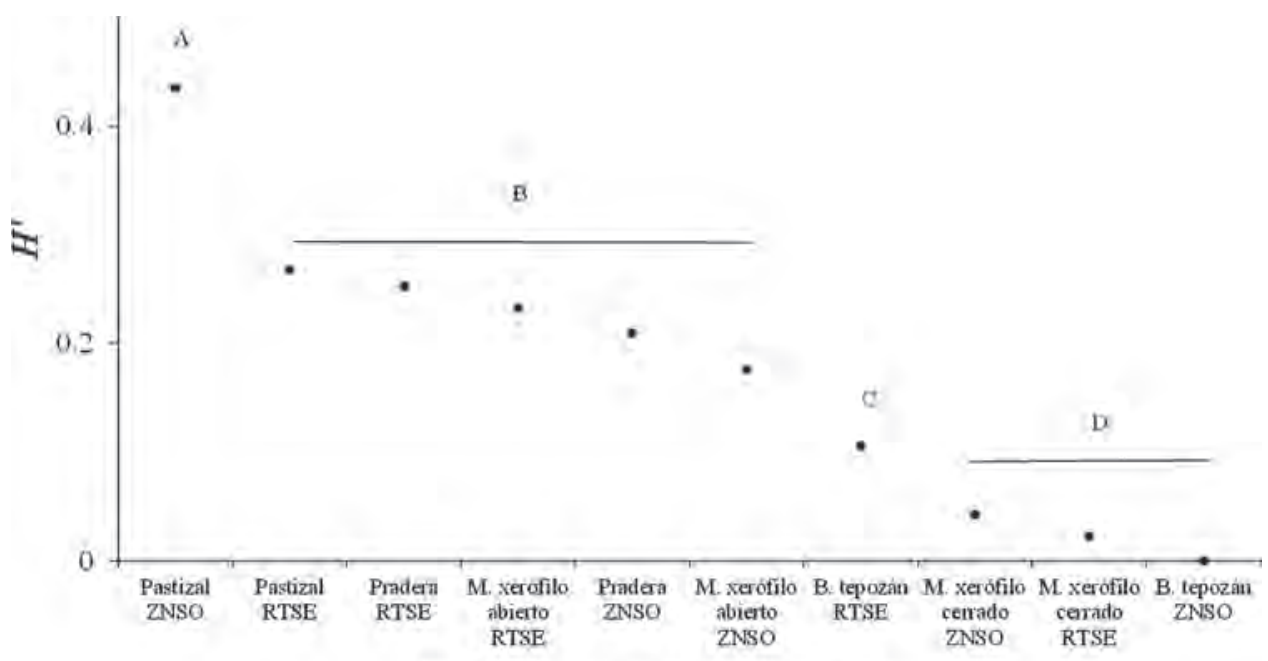

Sitios y comunidades vegetales

Figura 4. Variación de la diversidad de ortópteros calculada con base en el índice de Shannon-Wiener ( $H^{\prime}$ ) entre los tipos de vegetación de la Reserva Territorial Sureste (RTSE) y los de la Zona Núcleo Suroriente (ZNSO). Las letras diferentes denotan diferencias significativas según las pruebas de $t$ de Student con ajustes de Bonferroni para comparaciones múltiples.

tensión original del Pedregal de San Ángel fue de 80 km², y que a mediados del siglo XX se inició su fragmentación como resultado del proceso de urbanización debido al crecimiento de la población de la Ciudad de México. Lot \& Camarena (2009) indican que los principales proyectos de obra urbana que se construyeron en la década de 1950 en la zona del Pedregal fueron: el Fraccionamiento Jardines del Pedregal, el Anillo Periférico y la Ciudad Universitaria. Esta última se asentó sobre un terreno de 719 ha de las cuales el 33\% (237.3 ha) corresponden actualmente a la REPSA-CU y aproximadamente 199 ha pertenecen a la
Reserva Territorial Universitaria (RTU). Por su parte, las Áreas Verdes y la Infraestructura de Obra Civil (Edificios Universitarios) ocupan 150 y 73 ha respectivamente y las rutas de vialidad que comunican a los campi alcanzan 59.7 ha (Lot et al. 2012).

Las Zonas de Reserva Territorial Universitaria ostentan fragmentos relictuales de la comunidad vegetal que colonizó el Pedregal de San Ángel y particularmente, la vegetación de la RTSE brinda una muestra de la complejidad estructural de la vegetación y del sustrato del Pedregal. En tan sólo una extensión de 10.5 ha fue 
posible delimitar cuatro comunidades vegetales, así como determinar los ortópteros que las habitan. Una de las funciones de la RTSE es proyectar el crecimiento de la Universidad. En este trabajo existen evidencias que indican que posee un valor ambiental equiparable con la ZNSO. La REPSA-CU está decretada como Patrimonio Universitario Inalterable y debido a la estrecha colindancia que existe entre la RTSE y la ZNSO (Fig. 1), cualquier construcción en la RTSE acarreará efectos negativos sobre la ZNSO y también sobre las especies que la habitan.

Sólo 18 años después del inicio de la urbanización de la zona del Pedregal, Márquez-Mayaudón (1968) reportó la presencia de 26 especies de ortópteros, siendo notable en su trabajo el registro de Syntechna tarasca (Saussure, 1859) Phaneropteridae: Phaneropterinae, especie que en la actualidad no se ha detectado en diversos muestreos realizados sistemáticamente en los estratos de la vegetación de la REPSA-CU, así como en los meses de lluvias y secas; por lo que probablemente su población se extinguió en la localidad. Si bien es cierto que en este trabajo solamente se realizó un muestreo en octubre, este mes corresponde al de máxima abundancia de especies en floración y este factor potencializa la captura de los ortópteros herbívoros con mayor dominancia en los sitios.

Por otra parte, la diversidad de especies reportada en este trabajo (Cuadro 1) representa el 23.07\% del listado original de Márquez-Mayaudón (1968) y la presencia de Conocephalus (Xiphidion) ictus (Scudder, 1875) Tettigoniidae: Conocephalinae constituye un nuevo registro para esta reserva ecológica. Esta especie ocupó el $4^{\circ}$ lugar de importancia por su aporte porcentual a la composición de la comunidad ortopterológica en ambos sitios estudiados (RTSE y ZNSO) (Fig. 2). Para el caso de los ensíferos, es probable que el valor porcentual que se reporta en este trabajo se eleve si se implementara una colecta nocturna. Por ejemplo, Romero-Mata (com. pers.) reporta para la REPSA-CU la presencia de al menos dos especies de grillos arborícolas captados mediante un método de ubicación auditivo; no obstante, las restricciones de horario para el acceso en ambos sitios imposibilitaron la realización de otras técnicas de muestreo, incluyendo los trampeos nocturnos.

Los datos que mostramos en este trabajo probablemente constituyen una evidencia acerca de cómo el deterioro por urbanización de un Área Natural genera fragmentos aislados arrastrando en el proceso, a algunas especies a la extinción y a la vez promoviendo la colonización de otras.

De acuerdo con los registros de Barrientos-Lozano (2004), la subfamilia Conocephalinae tiene una amplia distribución geográfica y su presencia en el sitio de estu- dio puede ser una evidencia de la influencia Neotropical en la composición de la ortopterofauna de esta zona de la Ciudad de México.

Asimismo, el género Conocephalus, fue reportado con anterioridad como uno de los componentes principales de la ortopterofauna del Parque Nacional "El Cimatario", ubicado al sur de la ciudad de Querétaro (García-García 2006, García-García et al. 2010). Según los trabajos de García-García (2006) y García-García \& Fontana (2008), este género es emblemático de los pastizales secundarios conformados por P. clandestinum. Los datos de Powell (1977) sobre la biología de Conocephalus semivittatus en Nueva Zelanda señalan que la especie es muy exitosa en pastizales dominados por diversas gramíneas de los géneros Anthoxanthum, Bouteloua, Dactylis, Hierochloe, Lolium, Panicum y Stenotaphium; el autor atribuye el valor de adecuación del ortóptero al grosor de los tallos de estos pastos, pues ofrecen superficies óptimas para la oviposición.

Si bien es destacable el registro de $C$. ictus en el Sureste de la Ciudad Universitaria, esta especie está lejos de ser dominante en la ortopterofauna del lugar. En ambos sitios (RTSE y ZNSO) el ortóptero más conspicuo fue $S$. $p$. purpurascens ( $N=121$ individuos, en la RTSE y $N=$ 95 individuos, en la ZNSO); seguido en orden de importancia por $P$. nebrascensis ( $N=19$ individuos, en la RTSE y $N=17$ individuos, en la ZNSO).

De acuerdo con algunos resultados experimentales de Braschler et al. (2009), la fragmentación que se infringe a pastizales seminaturales provoca, a largo plazo (siete años), variaciones significativas de la densidad y la riqueza de especies de ortópteros, y a la vez provoca importantes respuestas diferenciales en la abundancia de las especies que componen a la comunidad. Los resultados experimentales de estos autores sugieren que ante el proceso de fragmentación de su hábitat, las poblaciones de ortópteros del suborden Ensifera están más propensas a experimentar extinciones locales, mientras que las del suborden Caelifera mantienen, en apariencia, invariable sus valores de densidad.

Nuestro trabajo ofrece resultados congruentes con los que reportó Braschler et al. (2009): (1) tras 64 años de modificaciones al Pedregal sugerimos la posibilidad de extinción local de Syntechna tarasca; (2) evidenciamos el registro de $C$. ictus y (3) reportamos como dominantes a dos ortópteros del suborden Caelifera: $S$. p. purpurascens y P. nebrascensis.

De acuerdo con las observaciones de Kevan (1977a, b), S. p. purpurascens es un ortóptero muy común y ampliamente distribuido en el Centro de México y, según Cano-Santana (1994), es el herbívoro más importante de la zona del Pedregal debido a la densidad que puede 
alcanzar su población (hasta 22.8 ind. $/ \mathrm{m}^{2}$ ). Adicionalmente, este ortóptero pirgomórfino se coloca en una posición ventajosa en la comunidad de ortópteros; existen evidencias de que $S$. $p$. purpurascens resulta favorecido al vivir en pastizales donde domina $P$. clandestinum ya que forma "monocultivos". En sitios dominados por este pasto, se encuentran ootecas y huevos en alta densidad (4.6 ootecas $/ \mathrm{m}^{2}$ y 185 huevos $/ \mathrm{m}^{2}$ ), las ootecas alcanzan mayor volumen en comparación con las que produce en otros sitios con matorral xerófilo dentro de la REPSA-CU $\left(0.56 \mathrm{~cm}^{3} \pm\right.$ e.e. 0.04$)$, las ootecas poseen una mayor cantidad de huevos en su interior ( $40.3 \pm 1.6$ huevos/ooteca) y además, estos son más grandes $(5.1 \mathrm{~mm} \pm 0.6)$ (Castellanos-Vargas 2001, Castellanos-Vargas \& Cano-Santana 2009); mientras que en comparación con algunos ortópteros melanóplinos únicamente ponen $15.6 \pm 5.68$ huevos por ooteca en promedio (CESAVEH 2004).

La densidad y diversidad de ortópteros fue muy baja en sitios con vegetación de fisonomía cerrada (como los bosquetes de tepozán y los matorrales xerófilos, ver Figs. 3 y 4), esto es debido a que los ortópteros prefieren los sitios con vegetación abierta, con altos niveles de insolación y predominada por hierbas pues constituyen su alimento (Rivera-García 2011). Particularmente S. p. purpurascens evade la presencia de sitios sombríos y fríos, sólo recurre al dosel de los bosquetes de tepozán para pernoctar, resguardarse de las bajas temperaturas del invierno y buscar el último alimento verde de la temporada de lluvias (I. Castellanos datos no publ.).

Por su parte, $P$. nebrascensis fue la especie con el segundo lugar de importancia en la composición de la ortopterofauna. Castellanos-Vargas (datos no publ.) observó entre 1998-1999 que esta especie presentaba una densidad poblacional de 10 ind. $/ \mathrm{m}^{2}$ en los pastizales de la REPSA-CU dominados por $P$. clandestinum y que su distribución se limitaba a los bordes de esta Reserva. En esta contribución se reportaron 5.5 ind. $/ \mathrm{m}^{2}$ y la distribución de la especie se observó en las praderas de la RTSE y la ZNSO (Cuadro 2).

Joern (1983) indica que $P$. nebrascensis es una especie muy abundante y común que habita en las praderas centrales de los condados de Nebraska. Aproximadamente un $25 \%$ de las poblaciones que estudió se componían de individuos alados, en tanto que el $75 \%$ restante eran ápteros. Joern (1983) discute que esta especie posee un alto valor de plasticidad fenotípica que le permite desarrollar individuos alados y ápteros. Este autor argumenta que dicha respuesta está condicionada por las variaciones ambientales que se registran en las localidades donde habita la especie.

Las investigaciones de Uvarov (1977), Joern \& Gaines (1990), Squitier \& Capinera (2002), Kang et al. (2007) y de Wysiecki et al. (2011) señalan que la variación interanual de la densidad poblacional de varias especies de acrídidos está influenciada por variaciones de la temperatura y la humedad en las localidades que habitan; y recientemente se ha probado que las variaciones globales del clima (como los eventos de "El Niño” y “La Niña”), y cuyos efectos se pueden extender por largos períodos de tiempo, hacen que algunos parámetros demográficos (como la natalidad y la mortalidad) afecten sensiblemente el tamaño de las poblaciones de diversas especies de ortópteros y a la vez determinen sus patrones de abundancia en la comunidad y del funcionamiento trófico del ecosistéma (Chambers \& Samways 1998, Stenseth et al. 2002, Hurrell et al. 2003, Hallett et al. 2004, Jonas \& Joern 2007, Borchard et al. 2013).

Durante los años con lluvias abundantes, la producción de biomasa fresca de las plantas es alta y estos factores favorecen la abundancia y diversidad de ortópteros en la comunidad (Mysterud et al. 2003, Hallett et al. 2004); mientras que cuando el periodo de sequía se prolonga por varios años, la productividad de las plantas es baja y, en el caso de algunos pastizales, la acumulación de la biomasa seca afecta negativamente a las poblaciones de ortópteros ya que el alimento es menos digerible debido a que posee altos contenidos de fibras, y a la vez favorece la recurrencia de incendios, los cuales carbonizan a los huevecillos de estos insectos que se encuentran el suelo en estado de diapausa (Chambers \& Samways 1998, Meyer et al. 2002, Mysterud et al. 2003, Hao \& Kang 2004).

La ZNSO fue significativamente más diversa que la RTSE por la presencia exclusiva de T. pallidipenis (Cuadro 2). Es probable que los ortópteros mantengan un constante movimiento entre ambos sitios, lo cual permitiría mantener valores similares de abundancia, así como un flujo génico dinámico en las poblaciones (Guzmán \& Confalonieri 2010). Camacho-Castillo (1999) documentó que los adultos de $S$. $p$. purpurascens son capaces de moverse en promedio $10.5 \mathrm{~m} \times$ semana en sitios planos conservados de la REPSA-CU y en la actualidad se desconoce el ámbito de movimiento para muchas de las especies encontradas en este trabajo.

La diversidad también varió significativamente entre los tipos de vegetación (Fig. 4) y el valor más alto correspondió a los sitios con pastizal dominado por $P$. clandestinum $\left(H_{\text {ZNSO }}^{\prime}=0.434\right.$ y $H_{\text {RTSE }}^{\prime}=0.267$, ver Fig. 4). Se ha documentado que los pastizales atípicos sudafricanos (como el caso de $P$. clandestinum) poseen un alto valor nutritivo (Fulkerson et al. 1998) probablemente, este sea un factor determinante que permite soportar una alta diversidad de ortópteros. Finalmente, según las observaciones de Mendoza \& Tovar (1996), la comunidad vegetal de la REPSA-CU se compone de una serie de "parches" 
en los que ortópteros buscan y seleccionan activamente el alimento que mejor satisface sus necesidades.

Agradecimientos. Los autores manifiestan su más sincero agradecimiento al M.C. Enrique Mariño de la Colección Nacional de Insectos del Instituto de Biología de la UNAM (CNIN-IBUNAM) por el interés mostrado durante la elaboración de este trabajo, así como por la asesoría para el trabajo de gabinete. Sonia Juárez, Ernesto Zavala y Marcos Flores colaboraron con su asistencia técnica durante la colecta del material ortopterológico. Marco Antonio Romero brindó apoyo y asistencia técnica para el manejo y mantenimiento del equipo de cómputo en el laboratorio. Yuriana Martínez Orea brindó apoyo con la traducción del resumen. Los comentarios de dos revisores anónimos enriquecieron sustancialmente este trabajo.

\section{LITERATURA CITADA}

Álvarez-Sánchez, F. J., Carabias, J., Meave, J., Moreno-Casasola, P., Nava, D., Rodríguez, F., Tovar, C. \& Valiente, A. 1982. Proyecto para la creación de una Reserva en el Pedregal de San Ángel: México, Laboratorio de Ecología, Facultad de Ciencias, Universidad Nacional Autónoma de México (UNAM), México 49 pp.

Angold, P. G., Sadler, J. P., Hill, M. O., Pullin, A., Rushton, S., Rushton, S., Small, E., Wood, B., Wadsworth, R., Sanderson, R. \& Thompson, K. 2006. Biodiversity in urban habitat patches. Science of the Total Environment, 360: 196-204.

Barrientos-Lozano, L. 2004. Orthoptera. In: Llorente J., Morrone, J. J., Yáñez, O. \& Vargas, I. (Comp.). Biodiversidad, taxonomía y biogeografía de artrópodos de México: hacia una síntesis de su conocimiento. Volumen IV. Comisión Nacional para el Conocimiento y Uso de la Biodiversidad (CONABIO) y Universidad Nacional Autónoma de México (UNAM), México. pp. 603-625.

Borchard, F., Schulte, A. M. \& Fartmann, T. 2013. Rapid response of Orthoptera to restoration of montane heathland. Biodiversity and Conservation, 22: 687-700.

Braschler, B., Marini, L., Thommen, G. H. \& Baur, B. 2009. Effects of small-scale grassland fragmentation and frequent mowing on population density and species diversity of orthopterans: a longterm study. Ecological Entomology, 34: 321-329.

Camacho-Castillo, E. 1999. Demografía y movilidad de Sphenarium purpurascens (Orthoptera: Pyrgomorphidae) en la Reserva del Pedregal de San Ángel, D. F. (México). Universidad Nacional Autónoma de México, Facultad de Ciencias. Tesis Profesional. 69 pp.

Camarena-Berruecos, P. 2010. Xerojardineria, guía para el diseño de los jardines de Ciudad Universitaria. Secretaría Ejecutiva REPSACoordinación de la Investigación Científica-Universidad Nacional Autónoma de México. 92 pp.

Cano-Santana, Z. 1994. Flujo de energía a través de Sphenarium purpurascens (Orthoptera: Acrididae) y productividad primaria neta aérea en una comunidad xerófita. Tesis Doctoral. Unidad Académica de los Ciclos Profesionales y Posgrado del Colegio de Ciencias y Humanidades / Centro de Ecología, Universidad Nacional Autónoma de México. 198 pp.

Cano-Santana, Z. \& Meave, J. 1996. Sucesión primaria en derrames volcánicos: el caso del Xitle. Ciencias, 41: 58-68.

Capinera, J. L., Scott, R. D. \& Walker, T. J. 2004. Field Guide to Grasshoppers, Katydids and Crickets of the United States. Cornell University Press, New York. 249 pp.

Carrillo-Trueba, C. 1995. El Pedregal de San Ángel. Universidad Nacional Autónoma de México. 177 pp.
Castellanos-Vargas, I. 2001. Ecología de la oviposición de Sphenarium purpurascens (Orthoptera: Pyrgomorphidae) en la Reserva del Pedregal de San Ángel, México, D. F. Universidad Nacional Autónoma de México, Facultad de Ciencias. Tesis Profesional. 115 pp.

Castellanos-Vargas, I. \& Cano-Santana, Z. 2009. Historia natural y ecología de Sphenarium purpurascens (Orthoptera: Pyrgomorphidae). In: Lot, A. \& Cano-Santana, Z. (Comp.). Biodiversidad del Ecosistema del Pedregal de San Ángel. Universidad Nacional Autónoma de México. pp. 337-346.

Castillo-Argüero, S., Martínez-Orea, Y., Romero-Romero, M. A., Guadarrama-Chávez, P., Núñez-Castillo, O., Sánchez-Gallén, I. \& Meave, J. 2007. La Reserva Ecológica del Pedregal de San Ángel. Aspectos florísticos y ecológicos. Universidad Nacional Autónoma de México, 294 pp.

Castillo-Argüero, S., Montes-Cartas, G., Romero-Romero, M., Martínez-Orea, Y., Guadarrama-Chávez, P., Sánchez-Gallén, I. \& Núñez-Castillo, O. 2004. Dinámica y conservación de la flora del matorral xerófilo de la Reserva Ecológica del Pedregal de San Ángel (D.F., México). Boletín de la Sociedad Botánica de México, 74: 51-75.

CESAVEH. 2004. Memoria del Curso-Taller de Identificación y Manejo del Chapulín. Comité Estatal de Sanidad Vegetal del Estado de Hidalgo. San Miguel Regla, Huasca de Ocampo, Hidalgo. 103 pp.

Chambers, B. Q. \& Samways, M. J. 1998. Grasshopper response to a 40-year experimental burning and mowing regime, with recommendations for invertebrate conservation management. Biodiversity and Conservation, 7: 985-1012.

Cherrill, A. 2010. Species richness of Orthoptera along gradients of agricultural intensification and urbanization. Journal of Orthoptera Research, 19: 293-301.

de Wysiecki, M. L., Arturi, M., Torrusio, S. \& Cigliano, M. M. 2011. Influence of weather variables and plant communities on grasshopper density in the Southern Pampas, Argentina. Journal of Insect Science, 11: available online: insectscience.org/11.109 (Fecha de consulta: 20 de Agosto de 2014).

Eades, D. C., Otte, D., Cigliano, M. M. \& Braun, H. 2013 Orthoptera Species File. Version 5.0/5.0. <http://Orthoptera.SpeciesFile. org > (Fecha de consulta: 19 de Agosto de 2013).

Enciso de la Vega, S. 1979. Las lavas del Pedregal de San Ángel. Ciencia y Desarrollo, 25: 89-93.

Fulkerson, W. J., Slack, K., Hennessy, D. W. \& Hough, G. M. 1998. Nutrients in ryegrass (Lolium spp.), white clover (Trifolium repens) and kikuyu (Pennisetum clandestinum) pastures in relation to season and stage of regrowth in a subtropical enviroment. Australian Journal of Experimental Agriculture, 38: 227-240.

García-García, P. L. 2006. Diversidad, fenología y asociación con el hábitat de los ortópteros epífitos del Parque Nacional "El Cimatario”, Querétaro. Tesis de Maestría. Colegio de Postgraduados, Montecillo, Estado de México. 82 pp.

García-García, P. L. \& Fontana, P. 2008. Guía para el reconocimiento y estudio de los chapulines del Parque Nacional "El Cimatario”, Querétaro. Gobierno del Estado de Querétaro, Secretaría de Desarrollo Agropecuario. 115 pp.

García-García, P. L., Fontana, P., Equihua Martínez, A., Sánchez Escudero, J., Valdez Carrasco, J. \& Cano-Santana, Z. 2010. Diversidad y Fenología de los Ortópteros del Parque Nacional El Cimatario, Querétaro. Southwestern Entomologist, 35: 317-329.

Gaston, K. J., Smith, R. M., Thompson, K. \& Warren, P. H. 2005. Urban domestic gardens (II): experimental tests of methods for 
increasing biodiversity. Biodiversity and Conservation, 14: 395413.

Guzmán, N. V. \& Confalonieri, V. A. 2010. The evolution of South American populations of Trimerotropis pallidipennis (Oedipodinae: Acrididae) revisited: dispersion routes and origin of chromosomal inversion dines. Journal of Orthoptera Research, 19: 253-260.

Hallett, T. B., Coulson, E. A., Clutton-Brock, T. H., Pemberton, J. M. \& Grenfell, B. 2004. Why large-scale climate indices seem to predict ecological processes better than local weather. Nature, 430: 71-75.

Hao, S.-G. \& Kang, L. 2004. Effects of temperature on the postdiapause embryonic development and the hatching time in three grasshopper species (Orthoptera: Acrididae). Journal of Applied Entomology, 128: 95-101.

Hortelano-Moncada, Y., Cervantes, F. A. \& Trejo, A. 2009. Mamíferos silvestres. In: Lot, A. \& Cano-Santana, Z. (Comp.). Biodiversidad del Ecosistema del Pedregal de San Ángel. Universidad Nacional Autónoma de México. pp. 277-293.

Hurrell, J. W., Kushnir, Y., Otterson, G. \& Visbeck, M. 2003. An overview of the North Atlantic Oscillation. In: Hurrell, J. W., Kushnir, Y., Otterson, G. \& Visbeck, M. (Comp.). The North Atlantic Oscillation: climatic significance and environmental impact. Vol. 134. Geophysical Monograph. American Geophysical Union, Washington, D.C. pp. 1-35.

Joern, A. 1983. Population biology of Phoetaliotes nebrascensis. Metaleptea, 6: 8-9.

Joern, A. \& Gaines, S. B. 1990. Population dynamics and regulation in grasshoppers. In: Chapman R. F. \& Joern, A. (Comp.). Biology of grasshoppers. Ed. Wiley, New York, pp. 415-482.

Jonas, J. L. \& Joern, A. 2007. Grasshopper (Orthoptera: Acrididae) communities respond to fire, bison grazing and weather in North American tallgrass prairie: a long-term study. Oecologia, 153: 699-711.

Kang, L., Han, X., Zhang, Z. \& Sun, O. J. 2007. Grassland ecosystems in China: review of current knowledge and research advancement. Philosophical Transactions of the Royal Society B, 362: 997-1008.

Kevan, D. K. 1977a. Ord. Orthoptera s. str. (Saltatoria-Caelifera) Subord. Acridodea Infraord. Acridomorpha Superfam. Acridoidea Fam. Pyrgomorphidae. Orthopterum Catalogus, 16: iv $+663+$ (7).

Kevan, D. K. 1977b. The American Pyrgomophidae. Revista de la Sociedad Entomológica Argentina, 36: 3-28.

Knight, T. M. \& Holt, R. D. 2005. Fire generates spatial gradients in herbivory: an example from a Florida Sandhill ecosystem. Ecology, 86: 587-593.

Lot, .A. \& Camarena, P. 2009. El Pedregal de San Ángel de la Ciudad de México: Reserva Ecológica Urbana de la Universidad Nacional. In: Lot, A. \& Cano-Santana, Z. (Comp.). Biodiversidad del Ecosistema del Pedregal de San Ángel. Universidad Nacional Autónoma de México. pp. 19-25.

Lot, A., Pérez, M., Gil., G., Rodríguez, S. \& Camarena, P. 2012. La Reserva Ecológica del Pedregal de San Ángel, Atlas de Riesgos. Universidad Nacional Autónoma de México. 51 pp.

Márquez-Mayaudón, C. 1968. Contribución al estudio de los ortópteros de México. IV. Ortópteros del Pedregal de San Ángel, Villa Obregón, D.F. Anales del Instituto de Biología, Universidad Nacional Autónoma de México, Serie Zoología, 39: 107-112.

Mendoza, P. C. \& Tovar, E. 1996. Ecología de forrajeo de Sphenarium purpurascens (Orthoptera: Acrididae) en la reserva del
Pedregal de San Ángel, México, D. F. Universidad Nacional Autónoma de México, Facultad de Ciencias. Tesis Profesional. $97 \mathrm{pp}$.

Meyer, C. K., Whiles, M. R. \& Charleton, R. E. 2002. Life history, secondary production, and ecosystem significance of acridid grasshoppers in annually burned and unburned tall grass prairie. American Entomologist, 48: 52-61.

Mysterud, A., Stenseth, N. C., Yoccoz, N. G., Ottersen, G. \& Langvatn, R. 2003. The response of terrestrial ecosystems to climate variability associated with the North Atlantic Oscillation. In: Hurrell, J. W., Kushnir, Y., Otterson, G. \& Visbeck, M. (Comp.). The North Atlantic Oscillation: climatic significance and environmental impact. Vol. 134. Geophysical Monograph. American Geophysical Union, Washington, D.C. pp. 235-262.

Ortega-Álvarez, R. \& MacGregor-Fors, I. 2009. Living in the big city: Effects of urban land-use on bird community structure, diversity, and composition. Landscape and Urban Planning, 90: 189-195.

Peralta, H. A. \& Prado, M. J. 2009. Los límites y la cartografía. In: Lot, A. \& Cano-Santana, Z. (Comp.). Biodiversidad del Ecosistema del Pedregal de San Ángel. Universidad Nacional Autónoma de México. pp. 27-42.

Powell, G. S. 1977. Notes on the longhorned grasshopper Conocephalus semivittatus in New Zealand Orthoptera: Tettigoniidae. New Zealand Entomologist, 6: 324-325.

Rivera-García, E. 2011. Taxonomía y bioecología de plagas de chapulín en el Norte de México. In: García-Gutiérrez C. \& LozanoGutierrez, J. (Comp.). Control biológico de plaga de chapulín en el Norte-Centro de México. Zacatecas, Zacatecas. México. 15-32.

Rueda, A. M. \& Cano, Z. 2009. Artropodofauna. In: Lot, A. \& CanoSantana, Z. (Comp.). Biodiversidad del Ecosistema del Pedregal de San Ángel. Universidad Nacional Autónoma de México. pp. 171-201.

Rzedowski, J. 1954. Vegetación del Pedregal de San Ángel (D.F., México). Anales de la Escuela Nacional de Ciencias Biológicas del Instituto Politécnico Nacional de México, 8: 59-129.

Rzedowski, J. 1978. Vegetación de México. Ed. Limusa, México, D. F. 432 pp.

Rzedowski, J. \& Rzedowski, G. C. 1979. Flora Fanerogámica del Valle de México. Vol. 1. Ed Continental, México, D. F. 403 pp.

Santibáñez-Andrade, G. 2005. Caracterización de la heterogeneidad ambiental en la Reserva del Pedregal de San Ángel. Universidad Nacional Autónoma de México, Facultad de Ciencias. Tesis Profesional. $67 \mathrm{pp}$.

Santibáñez-Andrade, G., Castillo-Argüero, S., Zavala-Hurtado, J. A., Martínez Orea, Y. \& Hernández Apolinar, M. 2009. La heterogeneidad ambiental en un matorral xerófilo. Boletín de la Sociedad Botánica de México, 85: 71-79.

Sokal, R. \& Rohlf, J. 1995. Biometry. 3ª Edición. Ed. W. H. Freeman, New York. 887 pp.

Solomon, G. \& Samways, M. J. 2006. Topographic heterogeneity plays a crucial role for grasshopper diversity in a southern African megabiodiversity hotspot. Biodiversity and Conservation, 15: 231-244.

Squitier, J. M. \& Capinera, J. L. 2002. Observations on the phenology of common Florida grasshoppers (Orthoptera: Acrididae). Florida Entomologist, 85: 227-234.

StatSoft, Inc. 2008. Electronic Statistics Textbook: Tulsa, Oklahoma, StatSoft Corporation http://www.statsoft.com/textbook (Fecha de consulta: 14 de Enero de 2014).

Stenseth, N. C., Mysterud, A., Ottersen, G., Hurrell, J. W., Chan, 
K. S. \& Lima, M. 2002. Ecological effects of climate fluctuations. Science, 297: 1292-1296.

Suárez, A., Camarena, P., Herrera, I. \& Lot, A. 2011. Infraestructura verde y corredores ecológicos de los pedregales: ecología urbana del sur de la Ciudad de México. Universidad Nacional Autónoma de México (UNAM), Instituto de Ciencia y Tecnología del Distrito Federal (ICyTDF), Secretaría Ejecutiva de la Reserva Ecológica del Pedregal de San Ángel (SEREPSA). México, $86 \mathrm{pp}$.

Uvarov, B. 1977. Grasshoppers and Locust. Vol. 2. Centre of Overseas Pest Research, London. 613 pp.

Valiente-Banuet, A. \& de Luna, E. 1990. Una lista florística actua- lizada para la Reserva del Pedregal de San Ángel, México, D. F. Acta Botanica Mexicana, 9: 13-30.

Whittaker, R. H. 1965. Vegetation of the Great Smoky Mountains. Ecological Monographs, 26: 1-80.

Wood, P. A. \& Samways, M. J. 1991. Landscape element pattern and continuity of butterfly flight paths in an ecologically landscaped botanic garden, Natal, South Africa. Biological Conservation, 58: 149-166.

Zar, J. H. 2010. Biostatistical analysis. Ed. Pearson Prentice-Hall, Upper Saddle River, New Jersey. 944 pp.

Zepeda, T. 1961. La República Mexicana Geografía y Atlas.6 $6^{a}$ Edición. Ed. Progreso, México, D. F. 160 pp. 